Virginia Commonwealth University

VCU Scholars Compass

1993

\title{
Homogeneous nucleation in supersaturated vapors of polar molecules: Acetonitrile, benzonitrile, nitromethane, and nitrobenzene
}

\author{
D. Wright \\ Virginia Commonwealth University \\ R. Caldwell \\ Virginia Commonwealth University \\ C. Moxely \\ Virginia Commonwealth University \\ M. S. El-Shall \\ Virginia Commonwealth University, mselshal@vcu.edu
}

Follow this and additional works at: http://scholarscompass.vcu.edu/chem_pubs

Part of the Chemistry Commons

Wright, D., Caldwell, R., \& Moxely, C., et al. Homogeneous nucleation in supersaturated vapors of polar molecules: Acetonitrile, benzonitrile, nitromethane, and nitrobenzene. The Journal of Chemical Physics, 98, 3356 (1993).

Copyright (C) 1993 American Institute of Physics.

\section{Downloaded from}

http://scholarscompass.vcu.edu/chem_pubs/49

This Article is brought to you for free and open access by the Dept. of Chemistry at VCU Scholars Compass. It has been accepted for inclusion in Chemistry Publications by an authorized administrator of VCU Scholars Compass. For more information, please contact libcompass@vcu.edu. 


\title{
Homogeneous nucleation in supersaturated vapors of polar molecules: Acetonitrile, benzonitrile, nitromethane, and nitrobenzene
}

\author{
D. Wright, R. Caldwell, C. Moxely, and M. S. El-Shalla) \\ Department of Chemistry, Virginia Commonwealth University, Richmond, Virginia 23284-2006
}

(Received 21 August 1992; accepted 26 October 1992)

\begin{abstract}
The critical supersaturations $\left(S_{c}\right)$ required for the homogeneous nucleation of acetonitrile, benzonitrile, nitromethane, and nitrobenzene vapors have been measured over wide temperature ranges using a thermal diffusion cloud chamber. At $T_{r}=0.44$, the experimental results are higher than the predictions of the classical nucleation theory by $20 \%\left(\mathrm{CH}_{3} \mathrm{NO}_{2}\right), 50 \%$ $\left(\mathrm{CH}_{3} \mathrm{CN}\right), 57 \%\left(\mathrm{C}_{6} \mathrm{H}_{5} \mathrm{NO}_{2}\right)$, and $112 \%\left(\mathrm{C}_{6} \mathrm{H}_{5} \mathrm{CN}\right)$. This trend correlates well with the dipole moments and to a lesser extent with the polarizabilities of these substances. Corresponding states of simple fluids and scaled nucleation law show that $\mathrm{CH}_{3} \mathrm{CN}$ and $\mathrm{CH}_{3} \mathrm{NO}_{2}$ have similar nucleation behavior which is different from that of $\mathrm{C}_{6} \mathrm{H}_{5} \mathrm{CN}$ and $\mathrm{C}_{6} \mathrm{H}_{5} \mathrm{NO}_{2}$. Correlations with other less polar substances are examined. The scaled law, with $\Omega$ (the excess surface entropy per molecule) determined from the temperature dependence of the bulk surface tension, gives a better description of the experimental results than the classical theory with the exception of nitromethane. The results suggest that the classical theory does not take proper account of the dipole-dipole interaction in calculating the free energy of the embryonic droplets.
\end{abstract}

\section{INTRODUCTION}

The homogeneous nucleation of a liquid from its vapor phase is a problem of current interest from both experimental and theoretical points of view. ${ }^{1-8}$ In particular, the study of homogeneous nucleation is important for a fundamental understanding of a wide range of problems including photoinduced, ion and binary nucleation, aerosol generation, cloud formation, and several other atmospheric processes. ${ }^{9}$ On the theoretical side, homogeneous nucleation presents an interesting problem where a unique interplay between thermodynamics and kinetics controls the overall process. ${ }^{2,3,5}$

A complete understanding of the nucleation phenomena is currently limited by two factors. First, the nucleus for homogeneous nucleation has never been observed directly in any experiment. Only the consequence of its presence, e.g., droplet formation, is observed. Even within this constraint, different experimental techniques such as cloud chambers (diffusion and expansion), supersonic nozzles and shock tubes provide measurements of droplet formation rates over different ranges of temperature and supersaturation that hardly overlap. ${ }^{10}$ This makes an experimental rate equation valid over a wide range of supersaturation and temperature a rather difficult task to achieve. Second, nucleation theory has advanced relatively slowly over the past 60 years since the development of the so called "classical nucleation theory" (CNT) by Becker, Doring, Frenkel, and Zeldovich. ${ }^{2,3,11}$ This theory is based on the capillarity approximation which treats the nucleus for condensation as a small fragment of a bulk liquid with the same macroscopic properties such as surface tension and

${ }^{\text {a) }}$ To whom correspondence should be addressed. density. Although the CNT has provided significant steps in qualitatively understanding the factors that control the formation of the new phase, it fails to provide a consistent molecular picture as well as quantitative predictions of the rate of nucleation as a function of temperature and supersaturation. $^{10,12}$ Several models have been suggested to improve the theory, but in most cases, they retain the capillarity approximation..$^{13-18}$ It appears that new approaches toward a complete molecular theory which avoid the CNT approximations have only been examined recently. ${ }^{19-22}$

In order to make progress, systematic nucleation studies are desirable. Our interest in this area has to do with the establishment of a more detailed understanding of the role of molecular properties such as dipole moment, polarizability, and structural factors on the homogeneous nucleation process. Our approach is based on studying the homogeneous nucleation of different classes of substances and relating the trends observed in nucleation thresholds and rates to the molecular properties of the systems under investigation. This approach is useful in a number of aspects. First, comparison among the series using some reference frame such as the CNT will identify the trends in nucleation resulting from a systematic variation of one molecular property. Second, because of the semiquantitative nature of the existing nucleation theories, the application of scaling and "corresponding states" to nucleation is necessary. By examining the temperature dependence of the homogeneous nucleation in dimensionless form, different compounds can be grouped according to some common nucleation behavior. Therefore, it will become possible to develop some new scaling laws for homogeneous nucleation which are not dependent on any particular form of theory. Third, the data for different classes of substances with a wide variety of molecular properties will serve as a 
good test for any new theory of nucleation. This is particularly important for some systems with complicated molecular structures such as long chain hydrocarbons and large polycyclic compounds or with specific molecular interactions such as hydrogen bonded and charge-transfer systems. These systems are particularly good candidates to test the validity of a molecular theory of nucleation. A successful molecular theory should be able to predict the nucleation behavior in the form of a single equation applicable to the entire spectrum of systems characterized by different types of interactions. It should not be biased toward simple fluids with uncomplicated structures. It should be able to account for such anomalies observed in "associated vapors" ${ }^{\text {"23-25 }}$ and highly polar liquids. ${ }^{26}$

The present paper deals with a comparative study of the homogeneous nucleation of a series of polar molecules, namely, acetonitrile, benzonitrile, nitromethane, and nitrobenzene where the dipole moment ranges from 3.5-4.4 D. In a previous report we presented results for the homogeneous nucleation of acetonitrile. ${ }^{26}$ Those results showed that the critical supersaturation required for the onset of nucleation, as measured in a diffusion cloud chamber, is higher than the prediction of the CNT by more than $40 \%$. This is an unexpected result given the good agreement found between the experimental values of critical supersaturations for many substances and the predictions of the $\mathrm{CNT}^{27,28}$ We attributed this discrepancy to an oriented dipole-dipole interaction within the curved surface of the embryonic droplets which causes the free energy barrier

TABLE I. Measured data for each experiment for benzonitrile. $T_{0}$, the temperature of the pool surface; $T_{1}$, the temperature of the top plate; $P_{b}$ the total pressure. Stability criteria: $P_{N} / P_{0}$, the pressure ratio; and $\rho_{1} / \rho_{0}$, the density ratio. $P_{0}$ is the equilibrium vapor pressure of the working fluid at $T_{0} ; \rho_{\mathrm{l}}$ and $\rho_{0}$ are the total density of the vapor-carrier gas mixture at $T_{1}$ and $T_{0}$, respectively.

\begin{tabular}{lccccc}
\hline \hline $\begin{array}{l}\text { Experiment } \\
\text { number }\end{array}$ & $T_{0}(\mathrm{~K})$ & $T_{1}(\mathrm{~K})$ & $P_{t}$ (Torr) & $P_{t} / P_{0}$ & $\rho_{1} / \rho_{0}$ \\
\hline 1 & 374.95 & 260.05 & 481.1 & 11.3 & 0.454 \\
2 & 381.15 & 265.15 & 493.2 & 9.08 & 0.389 \\
3 & 386.85 & 270.05 & 505.1 & 7.46 & 0.333 \\
4 & 393.45 & 275.25 & 519.3 & 5.98 & 0.280 \\
5 & 398.05 & 279.15 & 530.4 & 5.17 & 0.249 \\
6 & 402.35 & 282.85 & 541.2 & 4.52 & 0.222 \\
7 & 372.75 & 258.95 & 391.6 & 10.1 & 0.418 \\
8 & 378.55 & 263.95 & 402.6 & 8.22 & 0.359 \\
9 & 384.05 & 268.75 & 413.1 & 6.79 & 0.309 \\
10 & 390.55 & 274.65 & 424.9 & 5.45 & 0.259 \\
11 & 395.05 & 278.65 & 439.8 & 4.78 & 0.232 \\
12 & 399.35 & 282.65 & 443.2 & 4.12 & 0.205 \\
13 & 387.15 & 269.95 & 674.4 & 9.84 & 0.409 \\
14 & 392.45 & 274.45 & 682.7 & 8.16 & 0.356 \\
15 & 397.15 & 278.75 & 684.3 & 6.89 & 0.312 \\
16 & 402.55 & 282.85 & 682.0 & 5.66 & 0.267 \\
17 & 407.35 & 286.75 & 683.0 & 4.80 & 0.234 \\
\hline \hline
\end{tabular}

TABLE II. Measured data for nitromethane, see Table I for explanation.

\begin{tabular}{llllll}
\hline \hline $\begin{array}{l}\text { Experiment } \\
\text { number }\end{array}$ & $T_{0}(\mathrm{~K})$ & $T_{1}(\mathrm{~K})$ & $P_{t}$ (Torr) & $P_{d} / P_{0}$ & $\rho_{1} / \rho_{0}$ \\
\hline 1 & 317.45 & 242.15 & 509.0 & 5.89 & 0.393 \\
2 & 320.95 & 246.35 & 522.2 & 5.15 & 0.358 \\
3 & 324.85 & 249.25 & 534.8 & 4.44 & 0.322 \\
4 & 328.65 & 253.15 & 548.4 & 3.86 & 0.292 \\
5 & 333.35 & 257.45 & 567.6 & 3.27 & 0.259 \\
6 & 337.15 & 260.75 & 584.0 & 2.87 & 0.236 \\
7 & 341.25 & 265.15 & 608.4 & 2.54 & 0.217 \\
8 & 325.05 & 247.25 & 738.6 & 6.07 & 0.403 \\
9 & 327.05 & 249.55 & 749.0 & 5.64 & 0.383 \\
10 & 328.75 & 251.15 & 755.9 & 5.29 & 0.366 \\
11 & 335.65 & 258.75 & 792.7 & 4.15 & 0.309 \\
12 & 336.35 & 259.75 & 799.0 & 4.06 & 0.304 \\
13 & 339.95 & 262.65 & 820.5 & 3.61 & 0.280 \\
14 & 340.95 & 263.75 & 824.6 & 3.48 & 0.273 \\
15 & 344.05 & 266.75 & 844.2 & 3.15 & 0.255 \\
16 & 348.95 & 270.65 & 878.6 & 2.71 & 0.230 \\
17 & 352.05 & 273.05 & 901.7 & 2.48 & 0.215 \\
18 & 326.95 & 247.85 & 951.1 & 7.20 & 0.452 \\
19 & 330.15 & 250.85 & 968.4 & 6.39 & 0.418 \\
20 & 335.15 & 255.65 & 994.8 & 5.32 & 0.369 \\
21 & 335.05 & 256.05 & 996.6 & 5.35 & 0.320 \\
22 & 339.25 & 259.75 & 1023.2 & 4.62 & 0.335 \\
\hline \hline & & & & &
\end{tabular}

TABLE III. Measured data for nitrobenzene, see Table I for explanation.

\begin{tabular}{|c|c|c|c|c|c|}
\hline $\begin{array}{l}\text { Experiment } \\
\text { number }\end{array}$ & $T_{0}(\mathrm{~K})$ & $T_{1}(\mathrm{~K})$ & $P_{t}($ Torr $)$ & $P_{t} / P_{0}$ & $\rho_{1} / \rho_{c}$ \\
\hline 1 & 389.45 & 276.35 & 763.0 & 18.8 & 0.546 \\
\hline 2 & 400.35 & 285.75 & 792.8 & 12.5 & 0.416 \\
\hline 3 & 400.55 & 286.85 & 798.8 & 12.5 & 0.415 \\
\hline 4 & 405.35 & 291.05 & 815.5 & 10.6 & 0.368 \\
\hline 5 & 409.15 & 296.25 & 827.1 & 9.29 & 0.331 \\
\hline 6 & 410.35 & 295.25 & 833.0 & 8.94 & 0.324 \\
\hline 7 & 413.75 & 300.85 & 845.7 & 8.00 & 0.295 \\
\hline 8 & 421.55 & 306.65 & 872.5 & 6.21 & 0.245 \\
\hline 9 & 390.45 & 277.15 & 766.0 & 18.1 & 0.533 \\
\hline 10 & 388.75 & 276.95 & 763.6 & 19.3 & 0.554 \\
\hline 11 & 393.75 & 281.45 & 777.2 & 16.0 & 0.491 \\
\hline 12 & 394.95 & 281.75 & 781.6 & 15.3 & 0.478 \\
\hline 13 & 394.35 & 282.45 & 778.5 & 15.6 & 0.482 \\
\hline 14 & 395.45 & 283.45 & 782.8 & 15.0 & 0.470 \\
\hline 15 & 399.25 & 287.05 & 797.1 & 13.2 & 0.430 \\
\hline 16 & 399.35 & 288.25 & 794.3 & 13.1 & 0.424 \\
\hline 17 & 403.95 & 291.85 & 811.8 & 11.1 & 0.379 \\
\hline 18 & 404.95 & 292.65 & 816.3 & 10.8 & 0.370 \\
\hline 19 & 409.15 & 297.15 & 829.9 & 9.32 & 0.331 \\
\hline 20 & 408.75 & 295.35 & 828.9 & 9.45 & 0.336 \\
\hline 21 & 409.75 & 296.85 & 831.3 & 9.13 & 0.327 \\
\hline 22 & 416.05 & 301.75 & 851.0 & 7.39 & 0.278 \\
\hline 23 & 413.75 & 301.25 & 848.2 & 8.02 & 0.295 \\
\hline 24 & 418.15 & 305.35 & 864.9 & 6.96 & 0.264 \\
\hline 25 & 422.05 & 307.85 & 875.7 & 6.16 & 0.238 \\
\hline
\end{tabular}


for nucleation to increase relative to that for randomly oriented dipoles in weakly polar substances. In the present study we extend that investigation to other polar molecules including those containing a polarizable phenyl group as well. This choice of systems has the additional benefit of allowing one to assess the mutual effects of dipole moment and polarizability on the nucleation threshold.

In this paper we first present and discuss the experimental results obtained by using an upward thermal diffusion cloud chamber. Then we compare the results with the predictions of the CNT. We also analyze the data using a corresponding states correlation. This provides a comparison of the nucleation behavior of polar molecules with respect to a reference frame derived for simple fluids. Finally, we examine the application of a scaling law, which relates the onset of nucleation to the critical temperature and the excess surface entropy per molecule. We also use the scaling law to compare the experimental data of the studied compounds with the data from other less polar molecules.

\section{EXPERIMENT}

The present data were obtained using an upward thermal diffusion cloud chamber. The general description of this device and the details of its operation are available elsewhere. ${ }^{12,26,28}$ In this chamber the transport processes which produce supersaturation are one dimensional, and can be carefully computed so that the thermodynamic state is precisely known. The nucleation rates are chosen sufficiently small so that the growing droplets have negligible influence on the supersaturation. The onset of nucleation is determined visually by observing the forward scattering of light from drops falling through a horizontal $\mathrm{He}-\mathrm{Ne}$ laser beam positioned near the middle of the chamber. These drops originate near the elevation at which the maximum (peak) supersaturation occurs ( $\sim 0.7$ reduced height). A measurement consists of setting the temperature of both plates so that the chamber is barely nucleating at a rate of 1-3 drops $\mathrm{cm}^{-3} \mathrm{~s}^{-1}$. Under these conditions, measurements are made of the total pressure $P_{t}$ the temperature of the evaporating pool $T_{0}$, and the temperature of the upper plate $T_{1}$. The temperatures of both plates are then decreased (or increased) by about $5^{\circ} \mathrm{C}$ and the measurement repeated. To insure reproducibility each measurement was repeated at least three times. In order to eliminate the interference from ion nucleation resulting from cosmic ray or natural radioactive sources, the measurements are performed with a constant electric field of $40 \mathrm{~V} / \mathrm{cm}$ applied between the chamber plates. The temperature gradient required for a nucleation rate of $1-3$ drops $\mathrm{cm}^{-3} \mathrm{~s}^{-1}$ is usually smaller by $3-4$ deg in the absence of the applied field. This indicates a catalytic effect for ion nucleation in the studied polar vapors.

All chemicals were obtained from Aldrich with stated purity of at least $99 \%$ and were degassed repeatedly by the freeze-pump-thaw method and transferred to the cloud chamber through a vacuum line. The carrier gas was research grade helium ( $99.999 \%$ pure).

\section{RESULTS AND DISCUSSION}

The chamber parameters $T_{0}, T_{1}$, and $P_{t}$ under which a steady nucleation rate of $1-3$ drops $\mathrm{cm}^{-3} \mathrm{~s}^{-1}$ occurs for benzonitrile, nitromethane, and nitrobenzene are listed in Tables I, II, and III, respectively. The tables also contain the pressure ratio $P_{t} / P_{0}$ and the density ratio $\rho_{1} / \rho_{0}$ for each experiment, where $P_{0}$ is the equilibrium vapor pressure of the working fluid at $T_{0}$, and $\rho_{1}$ and $\rho_{0}$ are the total densities of the vapor-He mixtures at $T_{1}$ and $T_{0}$, respectively. The stability condition of the chamber is satisfied by the relation ${ }^{29,30}$

$$
P_{t} / P_{0} \ll \frac{1-M_{w} / M_{g}}{1-T_{0} / T_{1}},
$$

where $M_{w}$ and $M_{g}$ are the molecular weights of the working fluid and of the carrier gas, respectively. For the three compounds in this study the $\left(1-M_{w} / M_{g}\right) /\left(1-T_{0} / T_{1}\right)$ ratio ranges from $45-50$. As noted before, at lower pressure ratios (less than 2.5), the supersaturation depends on the amount of carrier gas used. ${ }^{30}$ At higher pressure ratios, convection in the chamber can easily take place. In all the experiments reported in Tables I-III, no visible convection is observed in the chamber and the total vapor density is a monotonically decreasing function of chamber height. Moreover, the density gradient $\rho_{1} / \rho_{0}$ is always less than 0.5 which ensures the absence of convection that may be caused by wall heat in the chamber. ${ }^{30}$

The thermophysical properties needed to solve the boundary value problem associated with heat and mass flux in the chamber to obtain the supersaturation and temperature profiles ${ }^{29}$ are given in Table IV. Most of these parameters are available in the literature as reported in Table IV. Only the binary diffusion coefficient in $\mathrm{He}\left(D_{a b}\right)$, the vapor viscosity $(\eta)$ and the vapor thermal conductivity $(\lambda)$ had to be calculated for all compounds. In addition, the ideal gas heat capacity $\left(C_{p}\right)$ for nitrobenzene is not available in the literature. It is calculated by Rihani and Doraiswamy's method of group contributions ${ }^{31}$ which gives an average error of $2.5 \% .^{32}$

$D_{a b}$ is calculated using the empirical correlation of Fuller et al. ${ }^{33}$ which results in an average error of $5 \% .{ }^{34}$ The vapor viscosity $(\eta)$ is calculated in two different ways using the method of Chung et al..$^{35}$ and the corresponding states relation of Lucas. ${ }^{36}$ The two calculated $\eta$ 's agree to within 7\%-9\%. The two-constant Sutherland equations reported in Table IV are based on the Lucas method. The vapor thermal conductivity is calculated using the Eucken correlation $^{37}$ and the method of Chung et al. ${ }^{38}$ The two 
TABLE IV. Thermophysical properties of acetonitrile, benzonitrile, nitromethane, and nitrobenzene. Values ${ }^{2}$ of $M$, the molecular weight; $D_{a b}$, the binary diffusion coefficient at $0^{\circ} \mathrm{C}$ and $1 \mathrm{~atm} ; s$, the exponent of its temperature dependence; $a$, the thermal diffusion factor; $\Delta H_{\text {vap, }}$ the enthalpy of vaporization at the normal boiling point in cal $/ \mathrm{mol} ; T_{c}$, the critical temperature in $\mathrm{K} ; T_{b}$, the normal boiling point in $\mathrm{K} ; P_{c}$, the critical pressure in bar; and expressions as functions of temperature for $P_{e}$, the equilibrium vapor pressure; $C_{p}$ the constant pressure specific heat of the vapor; $\sigma$, the surface tension; $d$, the density of the liquid; $\lambda$, the thermal conductivity of the vapor; and $\eta$, the viscosity of the vapor and $\eta_{1}$, the viscosity of the liquid.

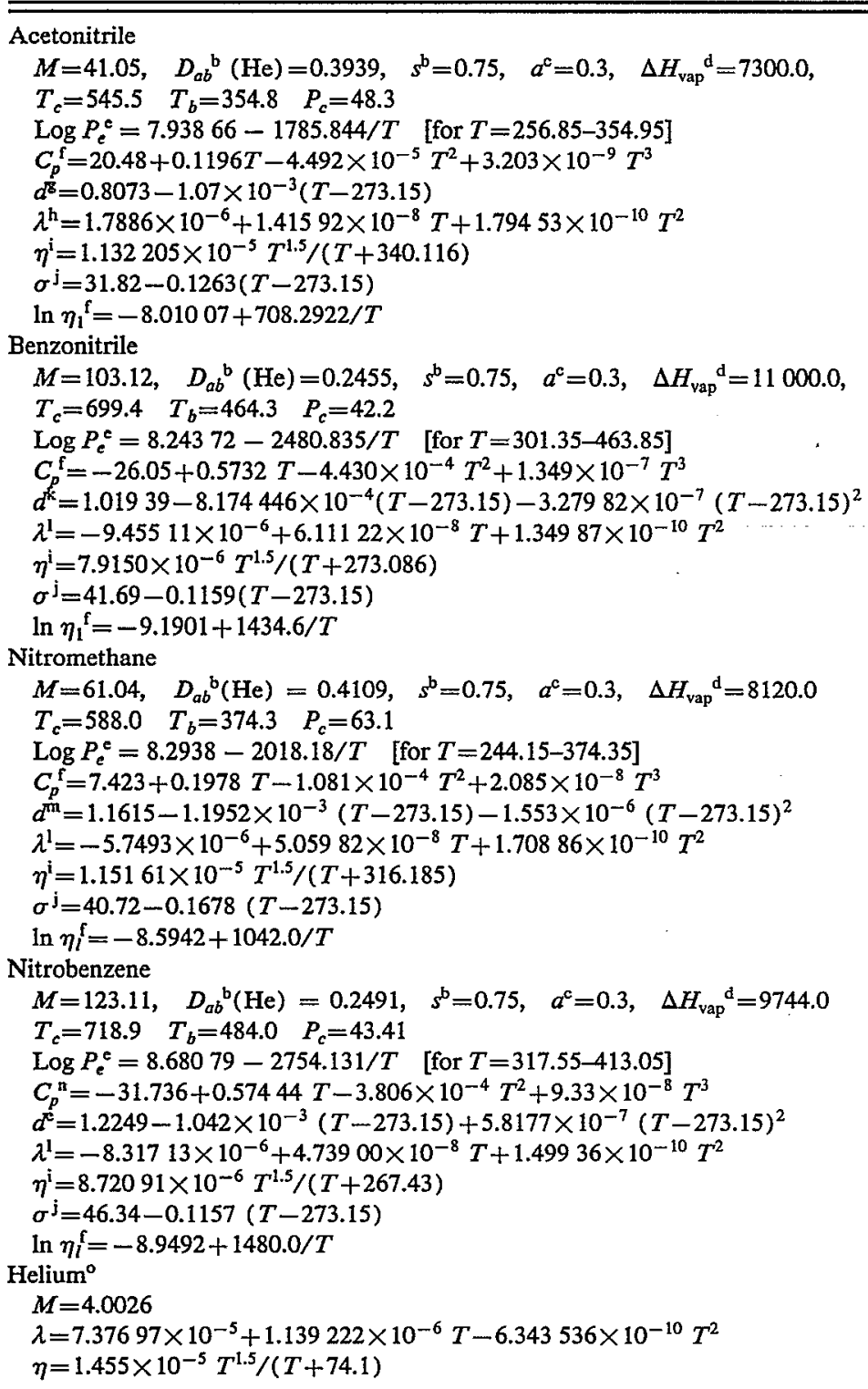

${ }^{2} M$ in $\mathrm{g} / \mathrm{mol} ; D_{a b}$ in $\mathrm{cm}^{2} / \mathrm{s} ; P_{e}$ in Torr; $C_{p}$ in $\mathrm{J} / \mathrm{mol} \mathrm{K} ; \sigma$ in dyn $/ \mathrm{cm} ; d$ in $\mathrm{g} / \mathrm{cm}^{3} ; \lambda$ in cal $/ \mathrm{cm} \mathrm{s} \mathrm{K;} \eta$ in Poise; $a$ and $s$ are unitless.

beference 33.

The thermal diffusion factor was approximated as $a=0.3$ by analogy to values for other mixtures found in K. E. Grew and T. L. Ibbs, Thermal Diffusion in Gases (Cambridge University, Cambridge, 1952), pp. $128-130$.

dReference 39.

${ }^{\text {'Reference } 40 .}$

Reference 34 .

BReference 41.

${ }^{\text {h}}$ Reference 42.

iReference 36.

${ }^{j}$ Reference 43.

${ }^{k}$ Reference 34, Eq. (3-11.1), p. 55.

'Reference 38.

meference 44.

${ }^{\mathrm{n}}$ Reference 31 .

${ }^{\circ}$ Reference 45. 
TABLE V. Dependence of the experimental and calculated supersaturations on the uncertainties in the estimated and literature parameters within the temperature range of the experiments, (lowest temp-highest temp).

\begin{tabular}{|c|c|c|c|c|c|}
\hline & \multirow[b]{2}{*}{$\%$} & \multicolumn{4}{|c|}{$\%$ change in $S_{c}$} \\
\hline Parameter & & $\mathrm{CH}_{3} \mathrm{CN}$ & $\mathrm{CH}_{3} \mathrm{NO}_{2}$ & $\mathrm{C}_{6} \mathrm{H}_{5} \mathrm{CN}$ & $\mathrm{C}_{6} \mathrm{H}_{5} \mathrm{NO}_{2}$ \\
\hline$a$ & 10 & $(0.28-0.30)$ & $(0.26-0.18)$ & $(0.43-0.28)$ & $(0.41-0.30)$ \\
\hline$s$ & 10 & $(0.83-0.32)$ & $(0.78-0.44)$ & $(1.20-0.78)$ & $(0.71-1.10)$ \\
\hline$\eta$ & 10 & $(1.0-0.17)$ & $(1.3-0.50)$ & $(1.60-1.50)$ & $(1.35-1.40)$ \\
\hline$C_{p}$ & 3 & $(0.10-0.87)$ & $(0.22-0.77)$ & $(0.15-0.56)$ & $(0.09-0.37)$ \\
\hline$P_{e}$ & 3 & $(0.44-0.17)$ & $(0.52-0.01)$ & $(0.54-0.32)$ & $(0.44-0.39)$ \\
\hline \multicolumn{6}{|c|}{ CNT calculations } \\
\hline$P_{e}$ & 3 & $(0.08-0.05)$ & $(0.11-0.07)$ & $(0.18-0.13)$ & $(0.18-0.13)$ \\
\hline$\sigma$ & 1 & $(2.3-1.5)$ & $(3.1-2.2)$ & $(4.9-3.7)$ & $(4.8-3.8)$ \\
\hline
\end{tabular}

methods agree within $4 \%-6 \%$. The temperature fits for $\lambda$ reported in Table IV are based on the Chung method.

The dependence of peak supersaturation on the uncertainties in the estimated parameters and the accuracy of the literature values is shown in Table V. Based on the estimated errors in the thermophysical properties and the uncertainty in measuring $T_{0}, T_{1}$, and $P_{t}$, we estimate the overall error in the experimentally determined supersaturation as $3 \%-5 \%$.

Examples of the dependence of supersaturation, temperature, total vapor density, equilibrium vapor pressure, and partial pressure on elevation within the chamber are given in Fig. 1. The temperature of the liquid film on the top plate is calculated using the film correction. ${ }^{29,30}$ Twenty-eight points around the peak supersaturation achieved in the chamber as a function of temperature are plotted for each experiment in Figs. 2-5 for acetonitrile, nitromethane, benzonitrile, and nitrobenzene, respectively. The envelope of each set of peaks represents the variation of the critical supersaturation $\left(S_{c}\right)$ for each compound with temperature. The results for acetonitrile shown in Fig. 2 are slightly different from the data reported in Ref. 26 due to the inclusion of the film correction in the present study and to a small error in solving the heat transport equations. The new data for acetonitrile confirm the conclusions reached in Ref. 26.

The prediction of the CNT for the dependence of $S_{c}$ on $T$ is computed from the equation

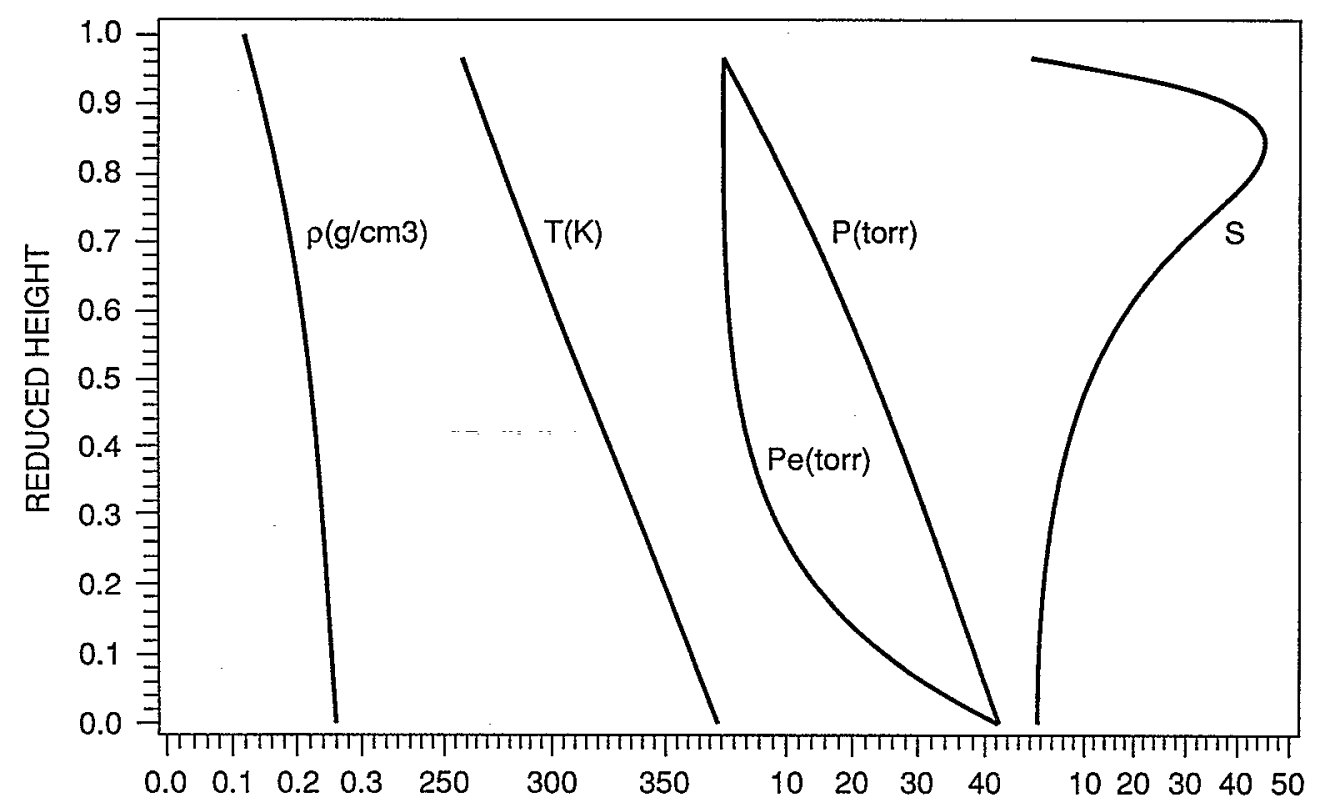

FIG. 1. Total vapor density, temperature, equilibrium vapor pressure, partial pressure, and supersaturation profiles calculated for the observed nucleation of 1-3 drops $\mathrm{cm}^{-3} \mathrm{~s}^{-1}$ for benzonitrile, $T_{0}=374.95 \mathrm{~K}, T_{1}=260.05 \mathrm{~K}$; and $P_{t}=481.1$ Torr. 


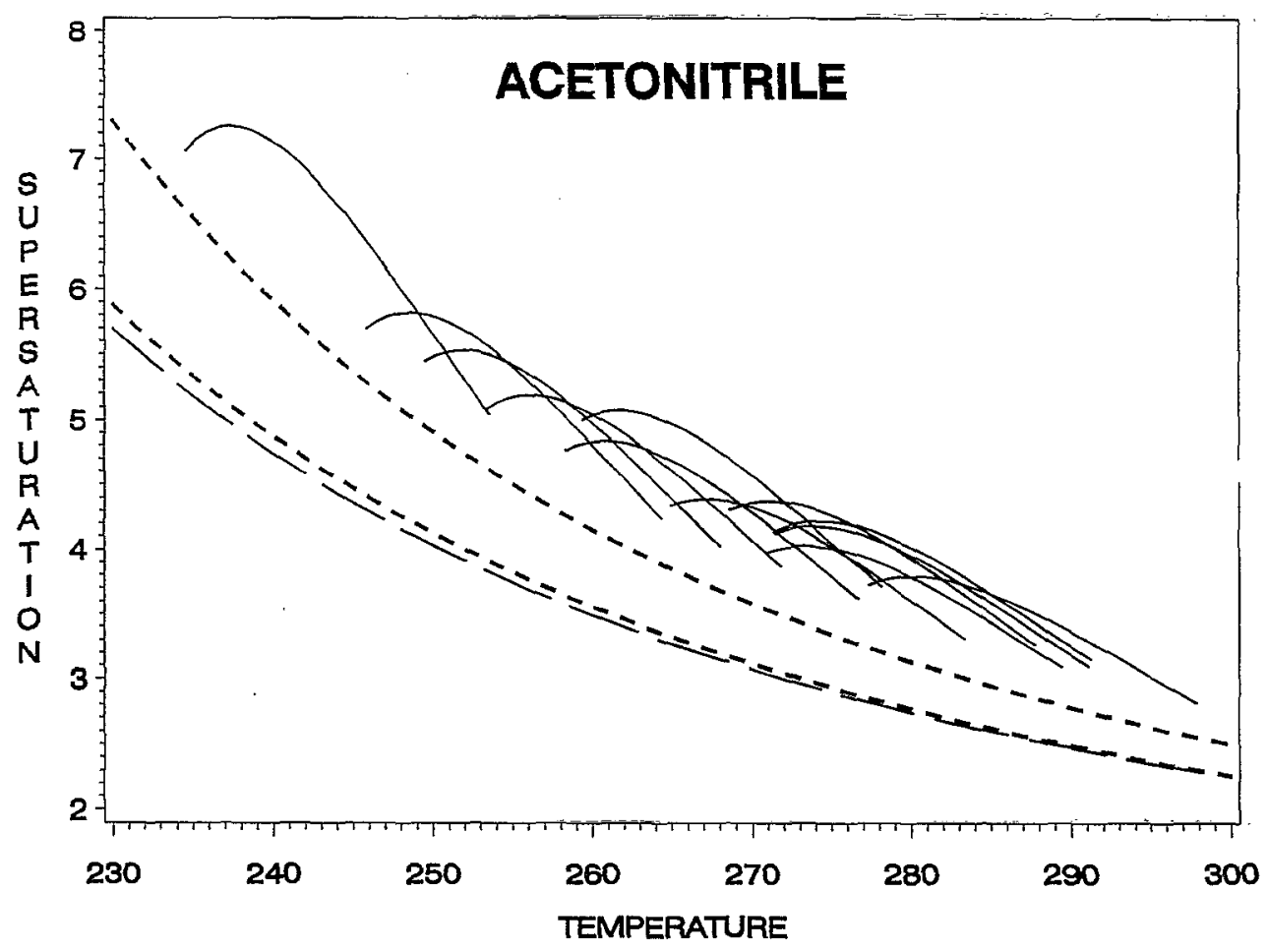

FIG. 2. Critical supersaturation vs temperature for acetonitrile. Dashed line represents theory. Envelope of solid curves represents experiment. Dotted lines are the results of the scaling law using $\Omega_{1}$ (lower dotted line) and $\Omega_{2}$ (upper dotted line). See the text for details.

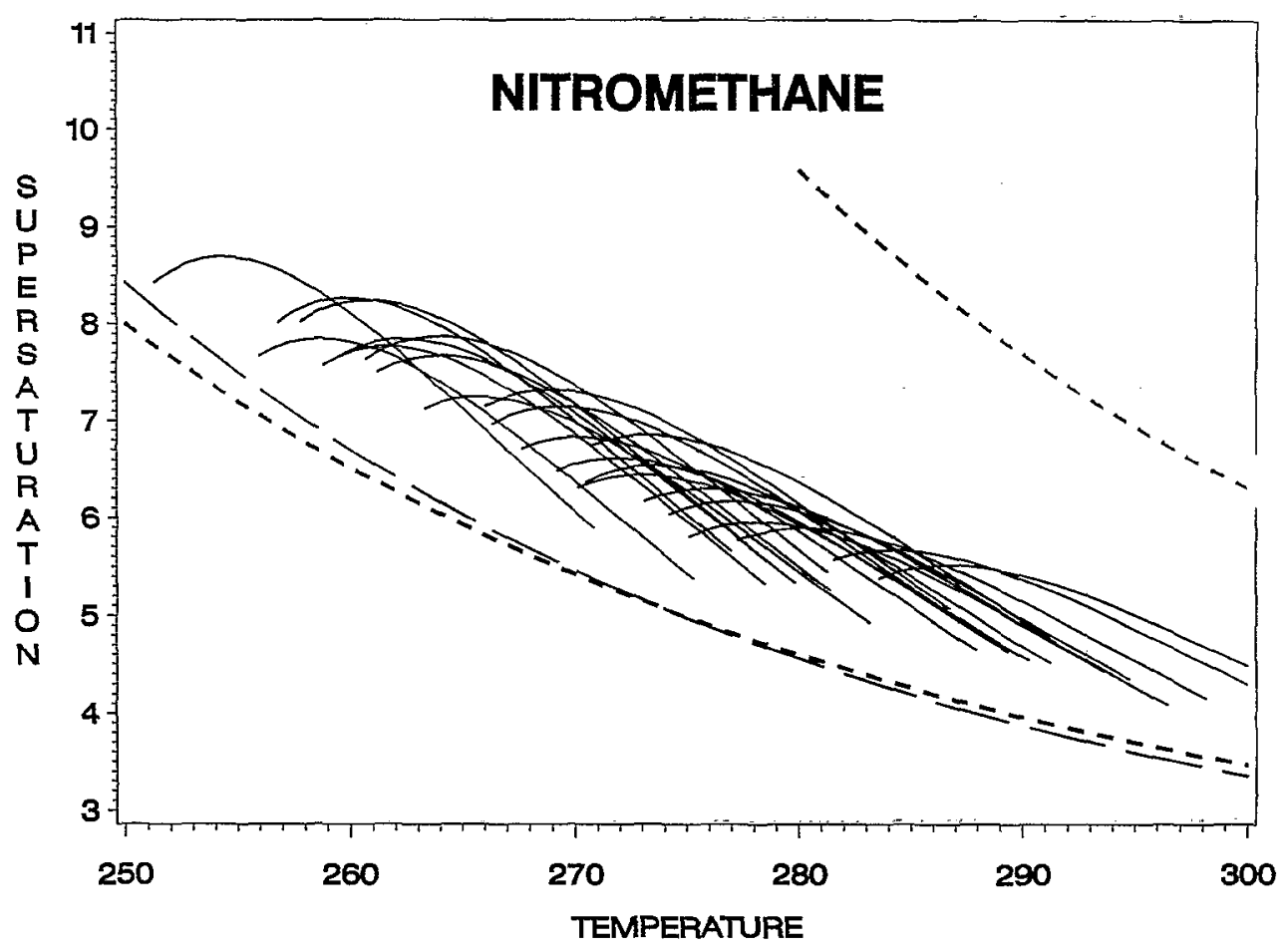

FIG. 3. Critical supersaturation vs temperature for nitromethane. Dashed line represents theory. Envelope of solid curves represents experiment. Dotted lines are the results of the scaling law using $\Omega_{1}$ (lower dotted line) and $\Omega_{2}$ (upper dotted line). See the text for details. 


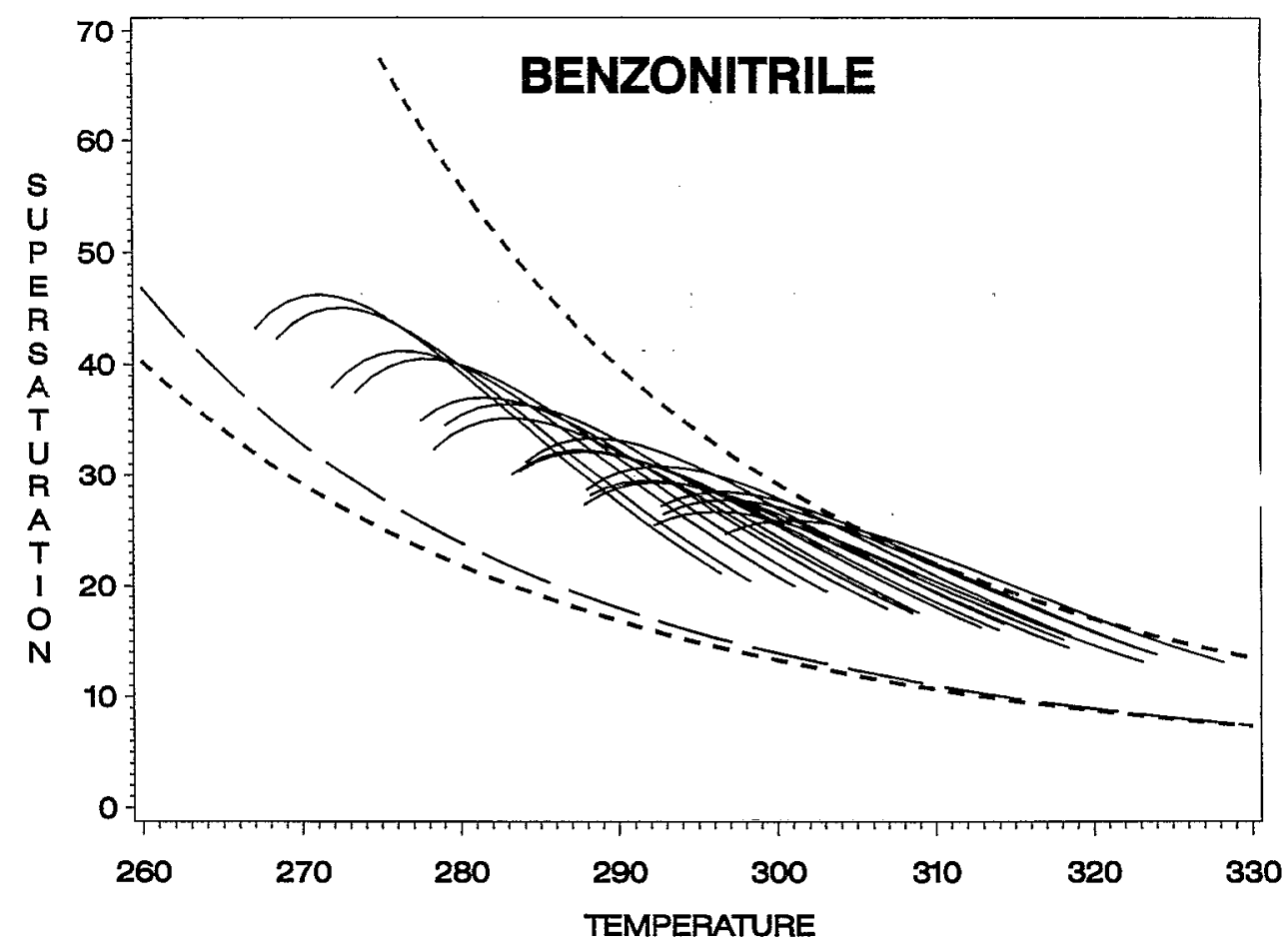

FIG. 4. Critical supersaturation vs temperature for benzonitrile. Dashed line represents theory. Envelope of solid curves represents experiment. Dotted lines are the results of the scaling law using $\Omega_{1}$ (lower dotted line) and $\Omega_{2}$ (upper dotted line). See the text for details.

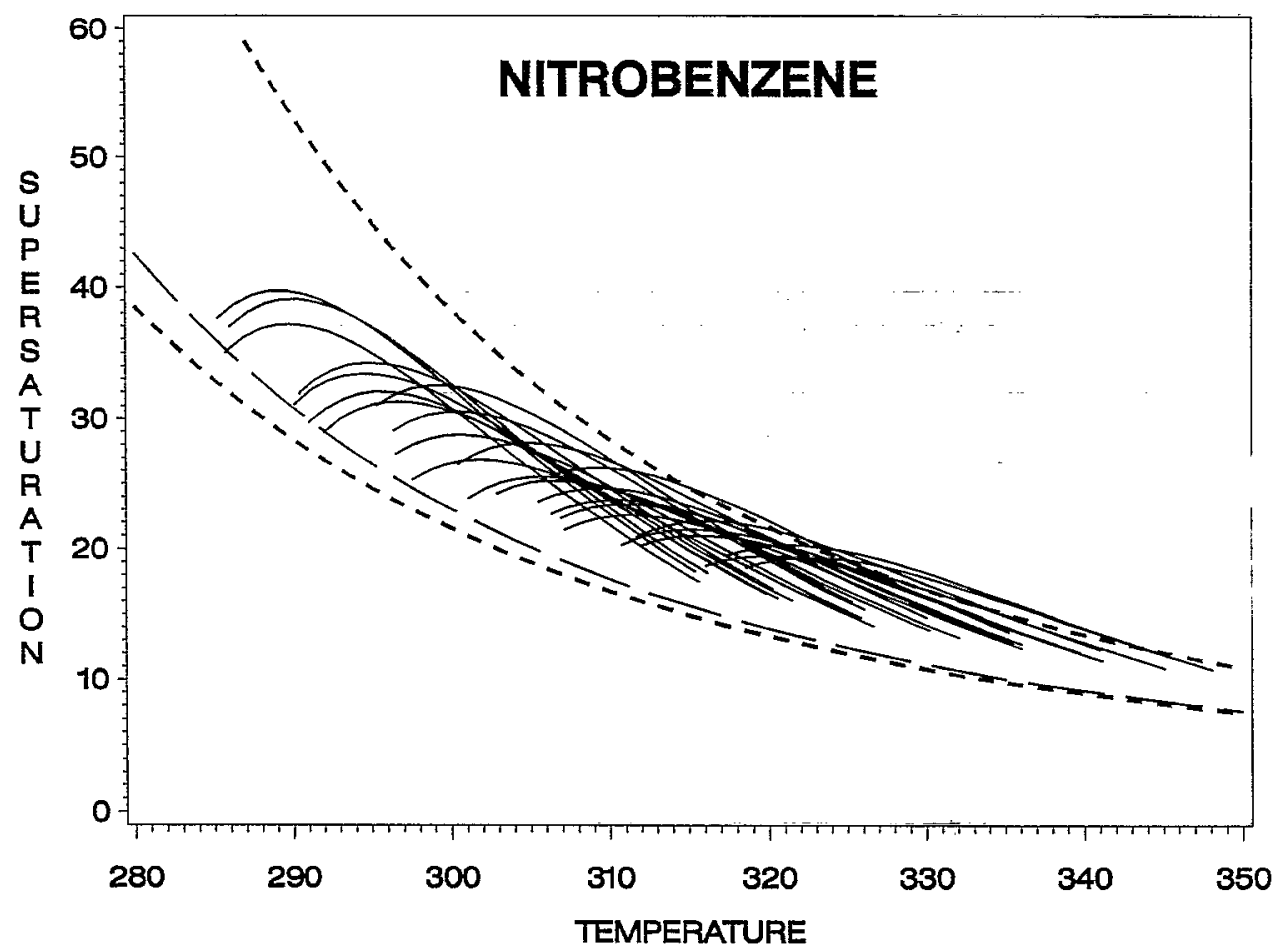

FIG. 5. Critical supersaturation vs temperature for nitrobenzene. Dashed line represents theory. Envelope of solid curves represents experiment. Dotted lines are the results of the scaling law using $\Omega_{1}$ (lower dotted line) and $\Omega_{2}$ (upper dotted line). See the text for details. 
TABLE VI. Comparison between experimental and calculated supersaturations at a given reduced temperature.

\begin{tabular}{|c|c|c|c|c|c|c|c|c|c|}
\hline & $T_{r}$ & $T$ & $\mu^{\mathbf{a}}$ & $\omega^{\mathrm{b}}$ & $\alpha^{\mathfrak{c}}$ & $S(\exp )$ & $S(\mathrm{CNT})$ & $\operatorname{Dev}(S)^{d}$ & $\Delta(\sigma)^{\mathrm{e}}$ \\
\hline $\mathrm{CH}_{3} \mathrm{CN}$ & 0.44 & 240.0 & 3.9 & 0.327 & 4.40 & 7.1 & 4.7 & $50 \%$ & $17 \%$ \\
\hline $\mathrm{C}_{6} \mathrm{H}_{5} \mathrm{CN}$ & 0.44 & 307.7 & 4.4 & 0.362 & 12.5 & 24.6 & 11.6 & $112 \%$ & $21 \%$ \\
\hline $\mathrm{CH}_{3} \mathrm{NO}_{2}$ & 0.44 & 258.7 & 3.5 & 0.310 & 4.95 & 8.3 & 6.9 & $20 \%$ & $7 \%$ \\
\hline $\mathrm{C}_{6} \mathrm{H}_{5} \mathrm{NO}_{2}$ & 0.44 & 316.3 & 4.25 & $0.435^{f}$ & 13.0 & 23.8 & 15.1 & $57 \%$ & $11 \%$ \\
\hline
\end{tabular}

Dipole moment in Debye taken from Refs. 34 and 46.

'Pitzer acentric parameter taken from Ref. 34 (Data Bank).

'Polarizability in $\mathrm{cm}^{3} \times 10^{24}$ taken from Ref. 46.

¿\% deviation between CNT supersaturations and the experimental values at $T_{r}=0.44$.

e\% increase in bulk surface tension required to restore agreement between experiment and the CNT.

Reference 34, p. 61.

$$
J=(q / d)\left(2 N_{A}^{3} \sigma M / \pi\right)^{1 / 2}\left(S P_{e} / R T\right)^{2} \exp \left(-W^{*} / R T\right),
$$

where $J$ is the rate of nucleation $\left(\mathrm{cm}^{-3} \mathrm{~s}^{-1}\right), q$ is a sticking coefficient and is set equal to $1, d$ is the liquid density, $N_{A}$ is Avogadro's number, $\sigma$ is the flat surface tension of the liquid, $M$ is the molecular weight, $S$ is the supersaturation ratio $\left(P / P_{e}\right)$, where $P$ is the pressure of the vapor, and $P_{e}$ is the equilibrium or "saturation" vapor pressure at the temperature of the vapor, $R$ is the gas constant and $T$ is the temperature of the vapor. The central quantity in the rate equation is the barrier height $W^{*}$ which is given by

$$
W^{*}=16 \pi N_{A} M^{2} \sigma^{3} / 3(d R T \ln S)^{2}
$$

By setting $J=1$ in Eq. (2) and using the literature values of the equilibrium vapor pressure, liquid density, and surface tension (as listed in Table IV), the dependence of $S_{c}$ on $T$ is obtained for each compound. The results are shown as the dashed curves in Figs. 2-5. The dependence of the $S_{c}$ predicted by the CNT on the accuracy of the parameters is shown in Table $\mathrm{V}$.

It is clear from the results in Figs. 2-5 and the effects of the uncertainties in the thermochemical properties shown in Table V, that the predictions of the CNT deviate strongly from the experimental results. However, the temperature dependence of the supersaturation is well reproduced by the theory as seen in Figs. 2-5 where the experimental supersaturations are virtually parallel, and significantly higher, than those predicted by the CNT. The magnitude of the discrepancy in $S_{c}$ is shown in Table VI where we compare the experimental and theoretical $S_{c}$ for all the investigated compounds at a given reduced temperature. We note that the discrepancy is larger for the more polarizable aromatic compounds relative to the methyl compounds. This trend is parallel to the size of the dipole moments and the polarizabilities of these substances as shown in Table VI. We also note that the cyano compounds show larger discrepancies relative to the analogous nitro compounds. This is clearly observed in the difference between benzonitrile and nitrobenzene despite the fact that the dipole moments are similar and the two compounds have equal polarizabilities. This may be explained in terms of a more localized charge in benzonitrile which results in a stronger orientation of the dipoles.

It is also interesting that the discrepancy between experiment and theory correlates rather well with the acen- tric parameter $\omega$ as shown in Table VI. This parameter is a measure of the increase in the entropy of vaporization over that of a simple fluid, and thus it reflects the contributions from dipole-dipole and dipole-induced dipole forces to the intermolecular potential energy. The relatively large $\omega$ for nitrobenzene compared to benzonitrile does not match the larger deviation observed for benzonitrile over nitrobenzene. This suggests that the localized dipole in benzonitrile might contribute significantly to the large deviation observed.

Up to this point we observed a strong systematic deviation from the predictions of the CNT for highly polar molecules. Since the CNT predicts the correct temperature dependence of $S_{c}$ for these systems, this raises the question as to how large a surface tension of the critical nuclei compared to the bulk value is necessary in order to restore agreement between the CNT and experiment. As shown in Table VI, this requires an increase in the bulk surface tension by $7 \%$ for $\mathrm{CH}_{3} \mathrm{NO}_{2}$ to $21 \%$ for $\mathrm{C}_{6} \mathrm{H}_{5} \mathrm{CN}$. This suggests that if the CNT is essentially correct, the embryonic droplets of highly polar molecules might be adequately described by larger surface tensions than their bulk values. The significance of the large dipole moments in increasing the surface tension of spherical droplets is shown by the strong correlation between $\mu$ and the required correction in the bulk surface tension. Therefore, the present results suggest that the CNT does not take proper account of the dipole-dipole interaction in calculating the free energy of the clusters.

\section{CORRESPONDING STATES CORRELATION}

Because of the approximate nature of the CNT, corresponding states relations and scaling laws are necessary in order to correlate trends in critical supersaturations with molecular properties. $\mathrm{McGraw}^{47}$ derived the following dimensionless form for the free energy maximum of nucleation, $W^{*}$,

$$
W^{*} / k_{B} T=282.3 G\left(T_{r}\right)(\log S)^{-2},
$$

where

$$
G\left(T_{r}\right)=\frac{T_{r}^{-3}\left(1-T_{r}\right)^{11 / 3}}{\left[1+0.75\left(1-T_{r}\right)+1.75\left(1-T_{r}\right)^{1 / 3}\right]^{2}} .
$$




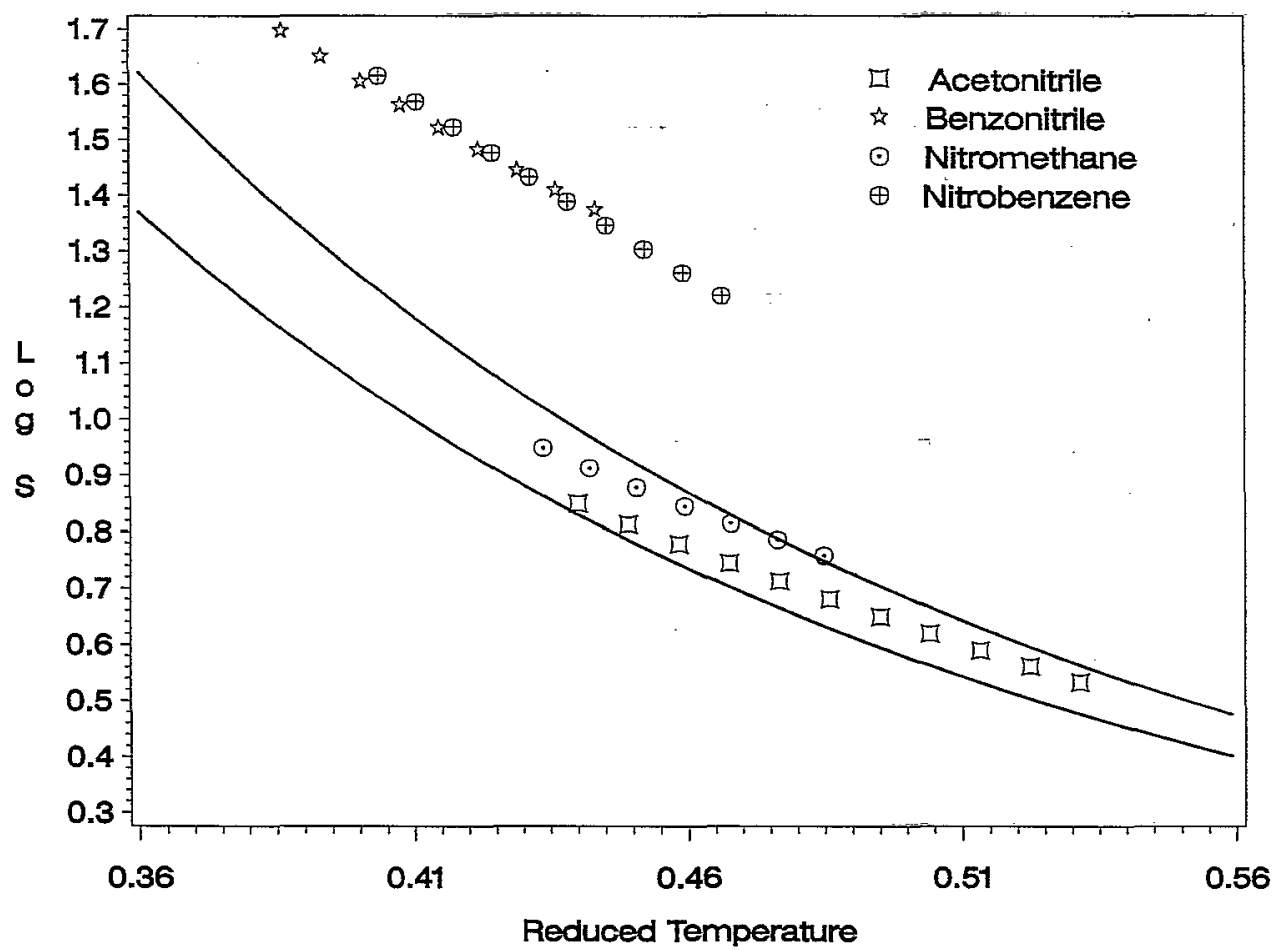

FIG. 6. Critical supersaturation vs reduced temperature. The lower and upper curves are for simple fluids and represent barrier heights [Eq. (4) in the text] of $70 \mathrm{kT}$ and $50 \mathrm{kT}$, respectively.

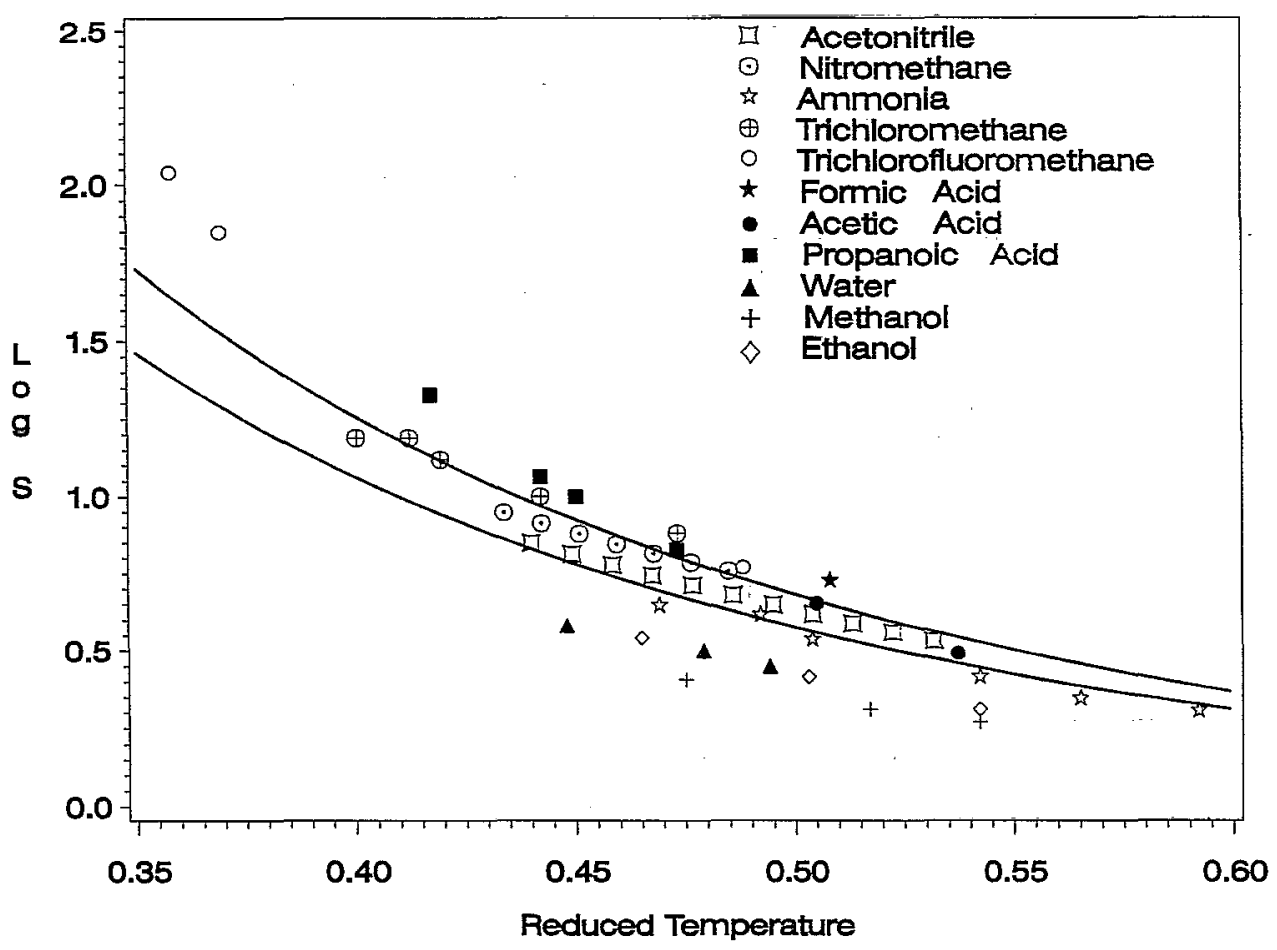

FIG. 7. Comparison between critical supersaturation vs reduced temperature for $\mathrm{CH}_{3} \mathrm{CN}$ and $\mathrm{CH}_{3} \mathrm{NO}_{2}$ with other polar compounds as indicated. The data for these compounds are obtained from Ref. 47. 


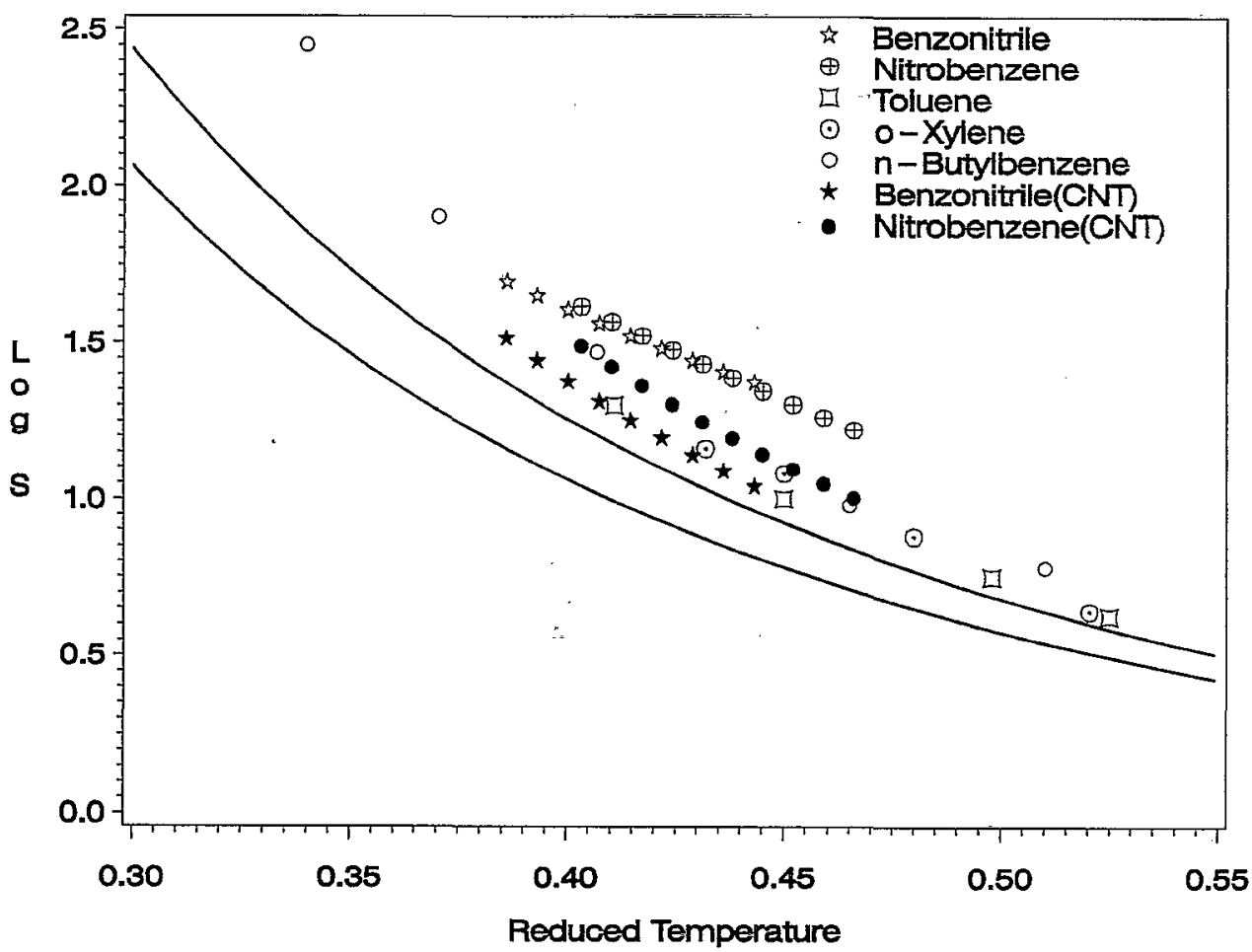

FIG. 8. Comparison between critical supersaturation vs reduced temperature for benzonitrile and nitrobenzene with other aromatic compounds as indicated. The data for these compounds are obtained from Ref. 47.

$k_{B}$ is Boltzmann's constant and $T_{r}=T / T_{c}$. Equation (4) is based on Guggenheim's empirical correlations of the surface tension and the number density of the heavier rare gases $^{48}$ and therefore is a good approximation for the reduced barrier height for simple fluids. For nucleation rates near unity $\left(J \sim 1 \mathrm{~cm}^{-3} \mathrm{~s}^{-1}\right) W^{*}$ lies between $50 k_{B} T$ and $70 k_{B} T^{47}$ The dependence of supersaturation on $T_{r}$ for simple fluids is shown in Fig. 6 as two solid curves for barrier heights of $50 k_{B} T$ and $70 k_{B} T$ along with the experimental $S_{c}$ values for the studied compounds. It is interesting to note that the compounds fall into two groups: the aromatics, benzonitrile and nitrobenzene; and the aliphatics, acetonitrile and nitromethane. Despite the large dipole moments, the latter group seems to conform well with the nucleation of simple fluids. Similar observations have been found for less polar fluids such as $\mathrm{NH}_{3}, \mathrm{CHCl}_{3}$, and $\mathrm{CCl}_{3} \mathrm{~F}$ and for associated vapors such as acetic and formic acids as shown in Fig. 7. However, the behavior of $\mathrm{CH}_{3} \mathrm{CN}$ and $\mathrm{CH}_{3} \mathrm{NO}_{2}$ is different from those polar compounds which form associated hydrogen bonded liquids such as water and alcohols which are characterized by smaller $S_{c}$ values compared to simple fluids. ${ }^{47}$ This suggests that the trend in the nucleation of $\mathrm{CH}_{3} \mathrm{CN}$ and $\mathrm{CH}_{3} \mathrm{NO}_{2}$ might be similar to that of associated vapors which also show higher critical supersaturations than the predictions of the CNT. This effect seems to cancel when the nucleation barrier is expressed in a dimensionless form and therefore $\mathrm{CH}_{3} \mathrm{CN}, \mathrm{CH}_{3} \mathrm{NO}_{2}$, and the carboxylic acids conform to the nucleation pattern of simple fluids which is higher than the CNT predictions for associated vapors. McGraw pointed out that the corresponding states of the nucleation thresholds incorporates the effect of vapor association in an approximate way. ${ }^{47}$ This is because $\epsilon_{0} / k_{B} T_{c}$ (where $\epsilon_{0}$ is the depth of the potential minimum) is a universal constant for simple fluids and hence any increase in $\epsilon_{0}$ due to association must be accompanied by an increase in $T_{c}$ to keep $\epsilon_{0} / k_{B} T_{c}$ constant. This leads to a reduction in $T_{r}$ which would mean an increase in $S_{c}$ of the associated vapors to conform to the pattern of simple fluids.

Figure 8 compares the critical supersaturations of benzonitrile and nitrobenzene with other aromatics having smaller dipole moments. Although all the compounds exhibit deviation from the simple fiuid pattern, benzonitrile and nitrobenzene show the largest deviation. The predictions of the CNT for benzonitrile and nitrobenzene are also shown in Fig. 8. Interestingly, these predictions correlate rather well with the experimental data for the less polar aromatics. This suggests that while the bulk surface tension used in the CNT might be appropriate to describe the clusters of weakly polar aromatics, it fails for highly polar clusters.

\section{SCALED NUCLEATION MODEL}

In this section, we analyze our results in terms of the scaled nucleation model developed by Hale. ${ }^{49-51}$ For a nucleation rate of $1 \mathrm{~cm}^{-3} \mathrm{~s}^{-1}$, the scaling law is expressed as

$$
\ln S_{c}=0.53 \Omega^{3 / 2}\left[\left(T_{c} / T\right)-1\right]^{3 / 2},
$$

where $\Omega$ is the excess surface entropy per molecule in the 
TABLE VII. Calculated and predicted (from the experimental supersaturations) values of $\Omega$.

\begin{tabular}{llll}
\hline \hline & $\Omega_{1}(T)^{\mathrm{a}}$ & $\Omega_{2}(T)^{\mathrm{b}}$ & $\Omega_{\exp }\left(T_{1}-T_{2}\right)^{\mathrm{c}}$ \\
\hline $\mathrm{CH}_{3} \mathrm{CN}$ & $1.63(270)$ & $1.76(270)$ & $1.88(240-300)$ \\
$\mathrm{CH}_{3} \mathrm{NO}_{2}$ & $1.84(270)$ & $2.39(270)$ & $1.99(255-290)$ \\
$\mathrm{C}_{6} \mathrm{H}_{5} \mathrm{CN}$ & $2.16(290)$ & $2.58(290)$ & $2.41(270-310)$ \\
$\mathrm{C}_{6} \mathrm{H}_{5} \mathrm{NO}_{2}$ & $2.31(310)$ & $2.59(310)$ & $2.49(290-340)$ \\
\hline \hline
\end{tabular}

${ }^{a}$ Calculated using Eq. (7) at a temperature $T(\mathrm{~K})$ which represents a mid-range of data.

${ }^{\mathrm{b}}$ Calculated using Eq. (8) at a temperature $T(\mathrm{~K})$ which represents a mid-range of data.

'Predicted from the experimental data using Eq. (6) and Fig. 9 at the temperature range of the data $\left(T_{1}-T_{2}\right)$.

cluster and can be approximated by the Eotvos constant, $K_{e}$, which is defined as ${ }^{52}$

$$
k_{B} N_{A}^{2 / 3} \Omega \approx K_{e}=v^{2 / 3} \sigma /\left(T_{c}-T\right),
$$

where $v$ is the liquid molar volume. Hale pointed out that a more reliable value for $\Omega$ can be obtained from the temperature dependence of the surface tension according to ${ }^{53}$

$$
\Omega=-\left[\frac{\partial \sigma}{\partial T}\right] /\left(k_{B} \rho^{2 / 3}\right),
$$

where $\rho$ is the liquid number density. In order to investigate the effect of the parameter $\Omega$ on the predictive ability of the scaling law, we used Eqs. (7) and (8) to calculate $\Omega$ for the studied compounds at a mid-range temperature of our data. The results are given in Table VII where we refer to $\Omega$ calculated from Eqs. (7) and (8) as $\Omega_{1}$ and $\Omega_{2}$, respectively. Using these parameters, we calculated the critical supersaturation as a function of temperature using Eq. (6). The results as shown in Figs. 2-5, indicate that the choice of $\Omega$ has a crucial effect on the calculated supersaturation. It is also evident that $\boldsymbol{\Omega}_{2}$ calculated from $\mathbf{E q}$. (8) does a relatively better job in predicting the supersaturation than $\Omega_{1}$ does. However, nitromethane shows an opposite trend where $\Omega_{1}$ gives results closer to the experimental data than does $\Omega_{2}$. We also note that the use of $\Omega_{1}$ in the scaling law gives rise to $S_{c}$ values comparable to the predictions of the CNT which underestimates the critical supersaturations for the highly polar compounds.

The measured supersaturations can be used to extract values for $\Omega$ from Eq. (6). Figure 9 exhibits plots of $\ln S_{c}$ vs $\left[\left(T_{c} / T\right)-1\right]^{3 / 2}$ based on the experimental values of $S_{c}$. The linearities of the plots are evident and from the slopes of the lines we calculated $\Omega$ values from our data. As expected, these values (listed in Table VII) are closer to $\Omega_{2}$ than to $\Omega_{1}$ with the exception of $\mathrm{CH}_{3} \mathrm{NO}_{2}$.

The most prominent feature in Fig. 9 is the grouping of the compounds in a similar fashion to that found with the corresponding states of simple fluids discussed in Sec. IV. The aromatic compounds benzonitrile and nitrobenzene require larger supersaturations than the aliphatics acetonitrile and nitromethane. Strong correlation within each group is also observed in Fig. 9. This is consistent with the similar polarizabilities of the compounds in each group as shown in Table VI. Figure 10 compares the results of ben-

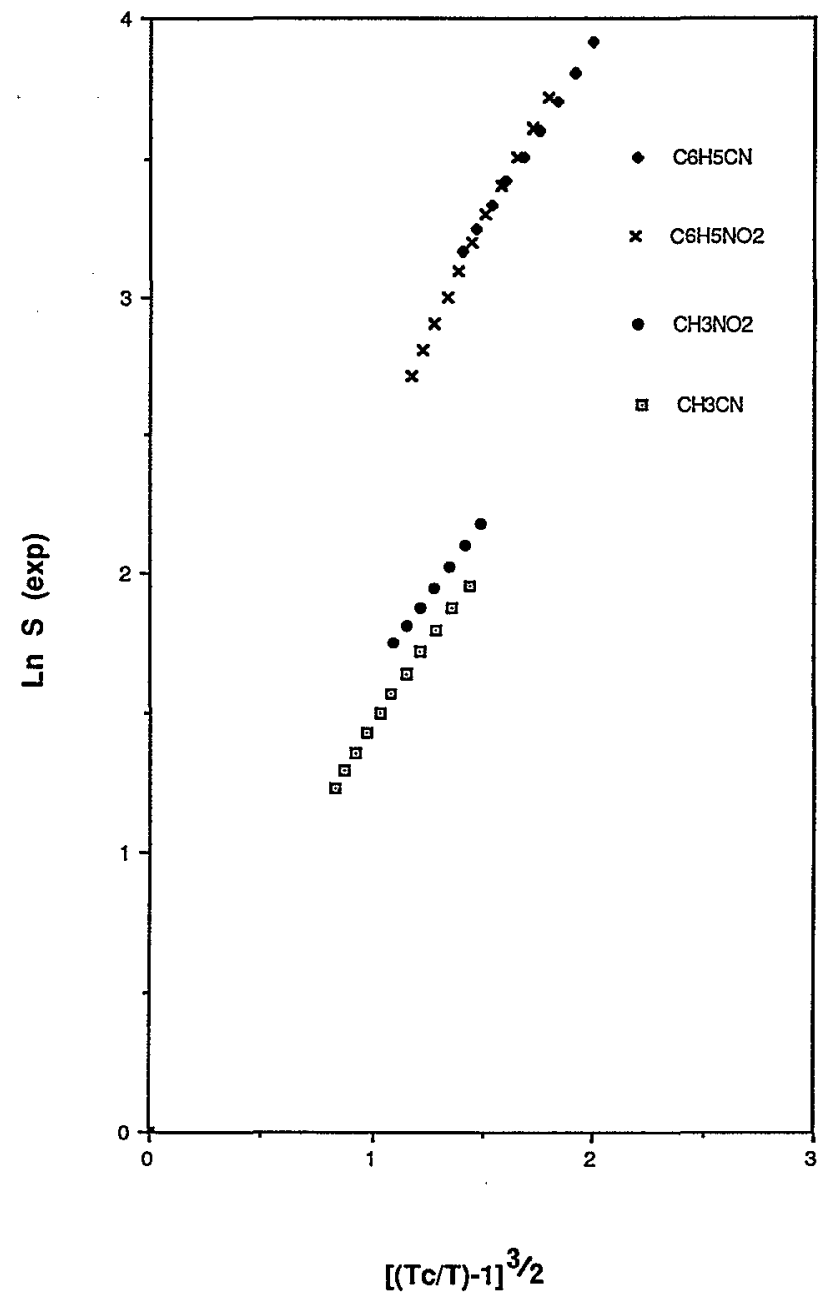

FIG. 9. $\left(\ln S_{c}\right)$ vs $\left[\left(T_{d} / T\right)-1\right]^{3 / 2}$ for the studied compounds.

zonitrile and nitrobenzene with those for less polar aromatic substances. Again, benzonitrile and nitrobenzene show larger supersaturations compared to the less polar aromatics which is the same trend observed in Fig. 8. Therefore, the characteristic nucleation behavior of the highly polar substances is clearly seen from the two scaled models.

\section{CONCLUSIONS}

The critical supersaturations required for the homogeneous nucleation of acetonitrile, benzonitrile, nitromethane and nitrobenzene have been measured. To our knowledge, this is the first reported measurement of the homogeneous nucleation of highly polar liquids, and while at this stage our data measures only the temperature dependence of the critical supersaturations, they are of sufficient accuracy to affirm several novel conclusions which we summarize as follows.

(1) The results show a significant disagreement with the predictions of the classical nucleation theory. Highly polar vapors are more stable with respect to condensation than those of weakly or nonpolar vapors which are well 


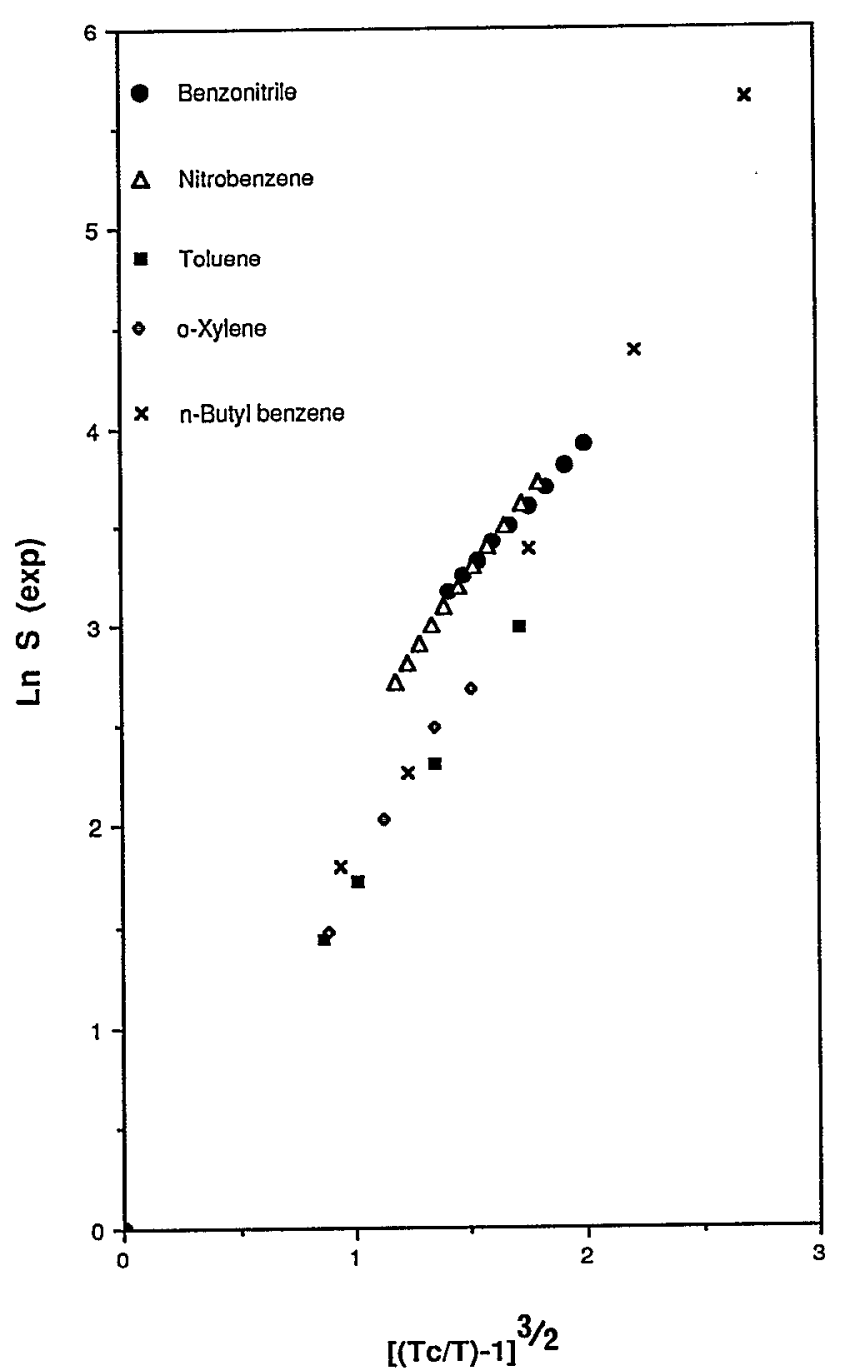

FIG. 10. Comparison between $\left(\ln S_{c}\right)$ vs $\left[\left(T_{d} / T\right)-1\right]^{3 / 2}$ plots for benzonitrile and nitrobenzene with similar plots for less polar aromatics. The data for the less polar aromatics are obtained from Ref. 47.

described by the CNT. The systematic deviations of the highly polar compounds from the CNT strongly correlate with the dipole moment and polarizability of the investigated molecules.

(2) Corresponding states and scaled nucleation models illustrate the similarity in the nucleation behavior of polar molecules with similar polarizability.

(3) The scaled law, with $\Omega$ extracted from the temperature derivative of the surface tension, describes the experimental results relatively better than the classical theory except for nitromethane. However, consistent and good agreement between the experimental results and the scaled model is lacking.

The results and analyses presented in this paper establish the characteristic behavior for the nucleation of highly polar liquids. However, these analyses do not provide a true explanation for such a phenomenon, and the theoretical basis of the observed behavior must be examined. It is quite possible that highly polar molecules exhibit a strong degree of association in the vapor phase which can influence both the kinetics and the thermodynamics of nucleation. ${ }^{54}$ It is also conceivable that large dipoles might align at the cluster surface and cause an increase in the surface free energy of the nucleus. ${ }^{55}$ The dipole alignments might be more conceivable in prolate spheriodal clusters. We address these questions in the paper that immediately follows the present one.

\section{ACKNOWLEDGMENTS}

Acknowledgment is made to the donors of the Petroleum Research Fund, administered by the American Chemical Society and to the Thomas F. and Kate Miller Jeffress Memorial Trust for the partial support of this research.

${ }^{1}$ Atmospheric Aerosols and Nucleation, edited by P. E. Wagner and G. Vali, Lecture Notes in Physics, Vol. 309 (Springer, Berlin, 1988).

${ }^{2}$ F. F. Abraham, Homogeneous Nucleation Theory (Academic, New York, 1974).

${ }^{3}$ Nucleation, edited by A. C. Zettlemoyer (Dekker, New York, 1969).

${ }^{4}$ P. Pal and M. R. Hoare, J. Phys. Chem. 91, 2474 (1987).

${ }^{5}$ K. Binder and M. H. Kalos, J. Stat. Phys. 22, 363 (1980).

${ }^{6}$ V. A. Shnidman and I. M. Fishman, Chem. Phys. Lett. 173, 331 (1990).

${ }^{7}$ C. F. Wilcox and S. H. Bauer, J. Chem. Phys. 94, 8302 (1991).

${ }^{8}$ B. Nowakowski and E. Ruckenstein, J. Chem. Phys. 94, 8487 (1991).

${ }^{9}$ See, for example, Aerosol Microphysics II: Chemical Physics of Microparticles, edited by W. H. Marlow (Springer, New York, 1982); T. Yamamoto and H. Hasegawa, Prog. Theor. Phys. 58, 816 (1977); N. H. Fletcher, The Physics of Rainclouds (Cambridge University, Cambridge, 1962).

${ }^{10}$ F. Peters and B. Paikert, J. Chem. Phys. 91, 5672 (1989).

${ }^{11}$ J. Frenkel, Kinetic Theory of Liquids (Dover, New York, 1955), Chap. 7.

${ }^{12}$ C. H. Hung, M. J. Krasnopler, and J. L. Katz, J. Chem. Phys. 90, 1856 (1989).

${ }^{13}$ J. Lothe and G. M. Pound, J. Chem. Phys. 48, 1849 (1968).

${ }^{14}$ H. Reiss, J. L. Katz, and R. E. Cohen, J. Chem. Phys. 48, 5553 (1968).

${ }^{15}$ J. S. Langer and L. A. Turski, Phys. Rev. A 8, 3230 (1973).

${ }^{16}$ J. L. Katz and M. D. Donohue, Adv. Chem. Phys. 40, 137 (1979).

${ }^{17}$ V. Ruth, J. P. Hirth, and G. M. Pound, J. Chem. Phys. 88, 7079 (1988).

${ }^{18}$ A. Dillman and G. E. A. Meier, Chem. Phys. Lett. 160, 71 (1989).

${ }^{19}$ D. W. Oxtoby and R. Evans, J. Chem. Phys. 89, 7521 (1988).

${ }^{20}$ X. C. Zeng and D. W. Oxtoby, J. Chem. Phys. 94, 4472 (1991); 95, 5940 (1991).

${ }^{21}$ H. Reiss, A. Tabazabeh, and J. Talbot, J. Chem. Phys. 92, 1266 (1990).

${ }^{22}$ H. M. Ellerby, C. L. Weakliem, and H. Reiss, J. Chem. Phys. 95, 9209 (1991).

${ }^{23}$ R. H. Heist, K. M. Colling, and C. S. Dupuis, J. Chem. Phys. 65, 5147 (1976).

${ }^{24}$ Y. G. Russell and R. H. Heist, J. Chem. Phys. 69, 3723 (1978).

${ }^{25}$ G. Agarwal and R. H. Heist, J. Chem. Phys. 73, 902, (1980).

${ }^{26}$ D. Wright, R. Caldwell, and M. S. El-Shall, Chem. Phys. Lett. 176, 46 (1991).

${ }^{27}$ M. S. El-Shall, J. Chem. Phys. 90, 6533 (1989), and references therein.

${ }^{28}$ M. S. El-Shall, J. Phys. Chem. 93, 8253 (1989).

${ }^{29}$ J. L. Katz, J. Chem Phys. 52, 4733 (1970).

${ }^{30}$ C. Becker, H. Reiss, and R. H. Heist, J. Chem. Phys. 68, 3585 (1978),

${ }^{31}$ D. N. Rihani and L. K. Doraiswamy, Ind. Eng. Chem. Fundam. 4, 17 (1965).

${ }^{32}$ R. C. Reid and T. K. Sherwood, The Properties of Gases and Liquids, 2nd ed. (McGraw-Hill, New York, 1966).

${ }^{33}$ E. N. Fuller, K. Enlet, and J. C. Giddings, J. Phys. Chem. 73, 3679 (1969).

${ }^{34}$ R. C. Reid, J. M. Prausnitz, and B. E. Boling, The Properties of Liquids and Gases, 4th ed. (McGraw-Hill, New York, 1987).

${ }^{35}$ Equation (9-4.9), Ref. 34, p. 396.

${ }^{36}$ Equation (9-4.15), Ref. 34, p. 400. 
${ }^{37}$ Equation (10-3.3), Ref. 34, p. 493.

${ }^{38}$ Equation (10-3.17), Ref. 34, p. 505.

${ }^{39}$ J. A. Dean, Handbook of Organic Chemistry (McGraw-Hill, New York, 1987).

${ }^{40}$ Handbook of Chemistry and Physics, 63rd ed., edited by R. C. Weast (Chemical Rubber Company, Boca Raton, 1982), D-203.

${ }^{41}$ R. R. Dreisbach, Physical Properties of Chemical Compounds, Advances in Chemistry Series, Vol. 2 (American Chemical Society, Washington, D.C., 1961), p. 392.

${ }^{42}$ D. Roy and G. Thodos, Ind. Eng. Chem. Fundam. 9, 71 (1970).

${ }^{43}$ J. J. Jasper, J. Phys. Chem. Ref. Data 1, 841 (1972).

${ }^{44} \mathrm{G}$. Korosi and E. Kovats, J. Chem. Eng. Data 26, 323 (1981); H. A. Berman and E. D. West, ibid. 12, 197 (1967).

${ }^{45}$ R. H. Heist and H. Reiss, J. Chem. Phys. 59, 15 (1973).

${ }^{46}$ L. G. Groves and S. Sugden, J. Chem. Soc. 1934, 1094; 1937, 158.

${ }^{47}$ R. McGraw, J. Chem. Phys. 75, 5514 (1981).
${ }^{48}$ E. A. Guggenheim, J. Chem. Phys. 13, 253 (1945).

${ }^{49}$ B. N. Hale, Phys. Rev. A 33, 4156 (1986).

${ }^{50}$ B. N. Hale, in Scaled Models for Nucleation, edited by P. E. Wagner and G. Vali, Lecture Notes in Physics, Vol. 309 (Springer, New York, 1988), pp. 323-349.

${ }^{51}$ B. N. Hale and P. Kemper, J. Chem. Phys. 91, 4314 (1989).

${ }^{52}$ A. Bondi, Physical Chemistry: Enriching Topics from Colloid and Surface Science, edited by H. V. Olphen and K. J. Mysels (Theorex, La Jolla, 1975), pp. 93-125.

${ }^{53}$ B. N. Hale and B. Kelly, Chem. Phys. Lett. 189, 100 (1992).

${ }^{54}$ J. L. Katz, H. Saltzburg, and H. Reiss, J. Colliod Interface Sci. 21, 560 (1966).

${ }^{55}$ F. F. Abraham, J. Chem. Phys. 50, 3977 (1969); Science 168, 833 (1970). 